\title{
An unavoidable bump: A meta-synthesis of psychotherapists' experiences of navigating therapy while pregnant
}

\author{
Carrie Way, ${ }^{1}$ Carolien Lamers, ${ }^{2}$ Renee Rickard ${ }^{2}$ \\ ${ }^{1}$ Betsi Cadwaladr University Health Board, North Wales; ${ }^{2}$ North Wales Clinical Psychology Programme, School of Psychology, \\ Bangor University, Wales, UK
}

\begin{abstract}
Despite psychotherapists' pregnancy being a common occurrence with recognised impacts for both clients and clinicians, there remains a dearth of empirical qualitative investigations into the lived experiences of these health professionals. This meta-synthesis therefore aims to generate novel insights and understandings of the experiences of 157 pregnant therapists by integrating the research findings of thirteen qualitative studies exploring the experiences of pregnant and newly post-partum psychotherapists. Utilising Noblit and Hare's (1988) meta-ethnographic approach, papers were analysed with a view to capturing shared experiences across studies, alongside points of divergence. Analysis led to the development of four key concepts: Identity Changes, Pregnancy necessitates Disclosure, Therapeutic Challenges and Guilt. Pregnancy was related to a multitude of personal and professional challenges, with the impact being most pronounced in the accounts of primiparous, child and trainee therapists. Key clinical implications include the need for continued exploration of the therapeutic impact of pregnancy in both supervision and therapy, revisions to supervisory working practices, prompt disclosure of therapist pregnancy and subsequent reductions to therapeutic fidelity. Future research directions are discussed within.
\end{abstract}

Correspondence: Carrie Way, Older Adults Psychology Service, Llys Dyfrig, Ffordd yr Orsedd, Llandudno LL30 1LA, North Wales, UK.

Tel.: 01492863360.

E-mail: carrie.way@wales.nhs.uk

Acknowledgements: The authors would like to thank the North Wales Clinical Psychology Programme for supporting the project.

Citation: Way, C., Lamers, C., \& Rickard, R. (2019). An unavoidable bump: a meta-synthesis of psychotherapists' experiences of navigating therapy while pregnant. Research in Psychotherapy: Psychopathology, Process and Outcome, 22(3), 450-463. doi: 10.4081/ripppo.2019.386

See online Appendix for additional materials.

Contributions: CW, CL, RR, conceptualisation of research question; $\mathrm{CW}$ acquisition of data, data analysis and drafting the manuscript; CL, RR, analysis triangulation; $\mathrm{CW}, \mathrm{CL}, \mathrm{RR}$, manuscript writing and reviewing.

Conflicts of interest: the authors declare no potential conflict of interest.

Funding: This research was conducted and funded as part of the first author's Clinical Psychology Doctorate at the North Wales Clinical Psychology Programme.

Received for publication: 11 March 2019.

Revision received: 9 September 2019.

Accepted for publication: 11 November 2019.

This work is licensed under a Creative Commons Attribution NonCommercial 4.0 License (CC BY-NC 4.0).

${ }^{\circ}$ Copyright: the Author(s), 2019

Licensee PAGEPress, Italy

Research in Psychotherapy:

Psychopathology, Process and Outcome 2019; 22:450-463

doi:10.4081/ripppo.2019.386
Key words: Pregnancy; psychotherapist; meta-synthesis; disclosure; supervision.

\section{Introduction}

Pregnancy represents a time of change for therapists who may be used to having unidirectional therapeutic relationships, which focus on clients' needs and issues. Traditionally, for psycho-dynamically orientated clinicians, the approach necessitates being a 'blank screen' onto which clients can project their fantasies. Regardless of therapeutic orientation, pregnancy represents a therapeutic transgression, literally and figuratively, drawing attention towards the therapists' personal life within the professional realm. Indeed, pregnancy in itself is indicative of further implicit disclosures, including the therapists' desire for a family and the likely presence of a personal and intimate relationship outside the therapeutic sphere.

Although pregnancy may be facilitative and represent an opportunity to model successful changes, for example in body image (Katzman, 1993) and life stage transition (Grossman, 1990), most research has indicated that pregnancy and maternity leave may interfere with clients' therapeutic progress. Pregnancy and impending maternity leave may signal the therapist's changing availability, priorities and the impending withdrawal of regular support. As such, most research exploring the impact of therapists' pregnancy for clients has documented increased risk-taking behaviours (Bassen, 1988), abandonment fears (Gibb, 2004), premature treatment termination (Berman, 1975) and non-attendance (Napoli, 1999), all of which may indicate that the therapists' pregnancy may be viewed as an 
unwanted 'intrusion' (Fenster, 1983) within the therapeutic space.

Despite pregnancy and maternity leave being a common occurrence within a largely female professional group and the potential deleterious impact on clients; there remains a paucity of professional guidance on how to navigate the clinical issues that may emerge in response to a therapist's pregnancy (British Psychological Society; personal communication, August 2017). It is therefore unclear how, and from whom, pregnant therapists may source their guidance.

Research indicates that there is often limited supervisory or organisational support available for pregnant therapists (Fenster, Phillips \& Rapoport, 1986; p.67), with studies reporting dismissive or even hostile attitudes towards pregnant colleagues (Baum \& Herring, 1975; Baum $\&$ Itzhaky, 2006). While such unsympathetic reactions may not be confined to clinical workplaces, the potential for pregnancy themes to encroach upon psychotherapists' working practices may be comparatively amplified due to the emphasis on the therapeutic alliance. Therefore, research identifying sources of professional support and guidance for expectant therapists may be particularly warranted.

While new prospective methods to explore the clinical impact of therapist pregnancy are emerging (Schmidt, Gastaud \& Ramires, 2018), published enquiries into pregnant and post-partum therapists' experiences of pregnancy have often lacked sufficient methodological rigour, using personal reflections and case vignettes to illustrate potential clinical issues (i.e., McGourty, 2013; Miller \& Giffin, 2019; Silverman, 2001; Whyte, 2004). Alternatively, studies purporting to use empirical methodologies have tended to summarise interview data without including raw transcript excerpts (i.e., Bassen, 1988; Baum \& Herring, 1975; Naparstek, 1976), thus limiting opportunities to assess study transparency and validity. Dyson and King observe that the content of published investigations rarely explores therapists' lived experiences, noting that the "literature has mainly focused on the reactions of clients to the therapist's pregnancy. Even when therapists do discuss their clients' reactions, they omit to comment on their personal experiences of pregnancy and their experiences with clients" (2008; p.28). Although such omissions may be due to study design or professional proclivities toward client experiences, this also signals a paucity of empirical understanding regarding therapists' lived experiences of pregnancy and motherhood.

To date, there have been limited attempts to pool the empirical findings of qualitative studies in this area. Indeed, a recent systematic review (Schmidt, Fiorini \& Ramires, 2015) of pregnancy in psychoanalytic psychotherapists identified only one empirical qualitative paper (Tonon, Romani \& Grossi, 2012). The present metasynthesis therefore looks to provide a comprehensive review of empirical, qualitative study findings relating to therapists' personal experiences of pregnancy and moth- erhood. In order to capture the greatest possible divergence and convergence of study findings, this paper explores the experiences of therapists employing a range of therapeutic orientations (as opposed to Schmidt et al.'s exclusive focus on psychoanalytic practitioners). Utilising Noblit and Hare's (1988) meta-ethnographic approach, this research looks to explore the personal experiences of pregnant and post-partum psychotherapists who provide long-term interventions to clients with an eclectic range of difficulties. This analytic process looks to identify and generate novel insights and understandings that can offer clinical guidance and support to therapists and their supervisors regarding the best practice and management of the common occurrence of pregnancy within the heavily female dominated profession of psychotherapy. By keeping the scope of inquiry broad, this meta-synthesis aims to capture a wide range of psychotherapist experiences.

The present research defines psychotherapists as mental health practitioners who offer interpersonal individual or group-based therapies for a range of client presentations, across various specialisms.

\section{Materials and Methods}

\section{Rationale for a meta-synthesis}

An emergent approach, meta-syntheses involve the interpretative synthesis of different qualitative sources of enquiry into a given phenomenon. Qualitative meta-syntheses therefore look to preserve the existing interpretations and meanings embedded within the original texts (Walsh \& Downe, 2005), whilst developing novel interpretations that emerge through consideration of the wider corpus of studies. To this end, meta-syntheses are concerned with deriving exploratory insights, rather than aggregative or descriptive accounts (Mohammed, Moles \& Chen, 2016).

Meta-syntheses are associated with multiple benefits including broadening understanding of key phenomena of interest, the development of new insights, theories and conceptual understandings and the identification of gaps in existing literature (Mohammed et al., 2016). To this end, meta-syntheses may prevent the need for multiple linear investigations into the same research area (Finfgeld, 2003). In summary, meta-syntheses aim to contribute methodological and theoretical advancements by expanding the existing research body, and may help to inform health policy, practice and research (Mohammed et al., 2016).

Although meta-syntheses have been described as the qualitative equivalent to quantitative meta-analyses (Schreiber, Crooks \& Stern, 1989; cited in Bondas \& Hall, 2007), there remains no 'recognised gold standard' for conducting meta-syntheses (Mohammed et al., 2016). The lack of consensus regarding the optimal approach to data selection and analysis reflects qualitative paradigms that view truths as subjective and multiply constructed (Finfgeld, 2003; Walsh \& Downe, 2005). Subsequently, it is 
considered acceptable that meta-syntheses may combine findings of qualitative studies using different methodologies in the pursuit of generating a holistic interpretative synthesis (Sandelowski \& Barroso, 2003a).

While researcher subjectivity is a common critique of all qualitative research, meta-synthesists may be at increased risk of interpreting data in accordance with their own views and perspectives as these researchers were not involved in the original data collection (Bondas \& Hall, 2007). It is therefore imperative that meta-synthesists look to enhance methodological transparency through processes such as researcher triangulation and personal reflexivity statements (Mohammed et al., 2016), which may in turn help to substantiate this nascent methodology (Finfgeld, 2003). To this end, it is important to acknowledge that the first author's interest in, and awareness of the issues related to therapist pregnancy have been shaped largely through personal experiences of navigating therapeutic work whilst pregnant with her first child. The present meta-synthesis therefore reflects the combined analysis of the thirteen research studies, interpreted through the perspectives of a pregnant, and then recently post-partum trainee clinical psychologist. Researcher triangulation was used throughout all stages of the metasynthesis to reduce potential bias (research scope, data collection, quality appraisal, data analysis, write up), however it is noted that the second and third authors also identify as mother-therapists.

\section{Data collection}

The electronic databases ProQuest ${ }^{1}$ and PsycARTICLES were systematically searched in September 2019 using the abstract domain. A Boolean search incorporating the following wildcard operators enabled the following key terms to be combined: "Expectant AND therapist" OR "pregnant AND therapist" OR "pregnancy AND therapist" OR "pregnant AND social work*" OR "pregnant AND psychologist".

Other databases (such as MEDLINE) and search terms such as 'psychiatrist' did not result in the discovery of additional articles and therefore were not included in the final search strategy. It was further decided not to combine the phrases 'pregnancy AND therapy' as this led to a high return of irrelevant articles, for example those focusing upon medical management or treatment trials for gestational conditions.

Considerable attempts were made to access articles and theses that were unobtainable via University access or inter-library loan. These included contacting authors directly via email $(n=6)$, ResearchGate $(n=14)$ and Uni-

ProQuest was utilized to concurrently search the following databases for articles published in English: PsycINFO; Social Science Premium Collection; ASSIA and Social Science Database. versity libraries $(n=6)$ to request article access. Thirteen responses and nine articles were gained, of which three were included in the final synthesis (Davis, 1997; KarivAgnon, 1988; Lyndon, 2013). A further four unavailable unpublished doctoral theses were purchased from ProQuest Dissertation Repository (Bashe, 1989; Byrnes, 2001; Fenster, 1983; Locker-Forman, 2005).

\section{Inclusion and exclusion criteria}

Study inclusion criteria for the meta-synthesis were as follows: i) published in English, ii) conducted qualitative interviews or focus groups with psychotherapists who have first-hand experience of providing therapy while pregnant, iii) exclusively reports the experiences of female psychotherapists, iv) where therapists are involved in established therapeutic relationships on which they can reflect on any potential impact of pregnancy, v) include sufficient raw data pertaining to the experiences of therapist participants, vi) raw data excerpts pertaining to the experiences of psychotherapists can be clearly differentiated from other clinical groups studied within the same research. Finally, vii) only research reporting original study findings were included, as opposed to texts that recount summaries of otherwise unavailable papers.

Studies were excluded based on the following criteria: i) quantitative design, ii) insufficient raw transcript excerpts, iii) lacking analytic interpretation of study findings, iv) undergraduate or masters dissertations (which may lack sufficient rigour and external ratification), v) research pertaining to the experiences of therapists solely offering brief interventions relating to issues of fertility and pregnancy (i.e., genetic counsellors).

As the objective of meta-syntheses is to retrieve all relevant studies as opposed to a composite sample (Barroso et al., 2003), unpublished doctoral theses were sought for holism. Whilst doctoral theses lack the blind peer-review process afforded to published articles, by virtue of the viva voce examination, doctoral theses have arguably gone through a similarly stringent process of peer review to attain the doctoral award. Indeed, it is argued that due to the quantity of data available for syntheses, unpublished dissertations may be preferable to peer-reviewed articles that may impose strict word limits and prevent extensive engagement with the data (Finfgeld, 2003). Therefore, the inclusion of unpublished grey literature may hold valuable insights alongside reducing publication bias (Beck, 2002b; cited in Bondas \& Hall, 2007).

\section{Search procedure and outcome}

The final search strategy identified thirteen studies for inclusion in the synthesis (Figure 1). After removing duplicates, all retrieved articles were screened by title. One hundred abstracts were read that were considered likely to be relevant to the topic of enquiry or where this was indeterminable from article title alone. Twenty-seven full 
text articles were retrieved and assessed for eligibility. As qualitative research is often poorly indexed (Mohammed et al., 2016) or unusually titled, full text articles were subject to snowball sampling of reference lists to identify other potentially relevant studies. This iterative approach led to the inclusion of an additional five studies.

The total procedure lead to the inclusion of three peer reviewed articles, one book chapter and nine unpublished doctoral theses. While the ratio of published papers appears low, many published meta-syntheses have reported analyses pertaining to four or fewer peer-reviewed qualitative studies (Hannes \& Macaitis, 2012).

As is evident from Figure 1, the majority of studies included in the meta-synthesis are unpublished doctoral theses. There may be several potential reasons why empirical research pertaining to therapist pregnancy may not reach formal publication. Firstly, it is acknowledged that the experiences of pregnant therapists may represent a niche research interest. During the final study selection process, it was noted that six authors of unpublished theses alluded to personal experience of pregnancy while conducting research. Speculatively, this may indicate that factors related to the experience of pregnancy and motherhood may increase interest in the research area; while simultaneously reducing the likelihood of subsequent publication.

\section{Quality appraisal of selected studies}

The most frequently utilised quality assessment tool for meta-syntheses (Hannes \& Macaitis, 2012), the Critical Appraisal Skills Programme (CASP, 2017) assesses the credibility, relevance and rigour of qualitative research. The checklist comprises ten aspects of quality assurance, the presence of which were subsequently endorsed, refuted or queried by the first author and independently triangulated by the other authors, see Table 1 .

According to the CASP, the quality of most studies was considered adequate, with no notable differences in quality according to when research was conducted, or between published and unpublished works. Indeed, the only study appraised as endorsing all ten quality aspects was an unpublished work (Davis, 1997). However, the majority of studies were found to insufficiently consider two key quality areas: a critical examination of the researchers' own position (achieved by only four studies: Davis, 1997; Grossman, 1990; Locker-Forman, 2005; Zackson, 2012) and although all studies alluded to consideration of ethical safeguards, this was only comprehensively documented in three papers (Davis, 1997; Lyndon, 2013; McCluskey, 2017).

The oldest eight studies (1983-2005) did not specify using particular qualitative approaches, which may reflect the emergence of qualitative methodologies over time.

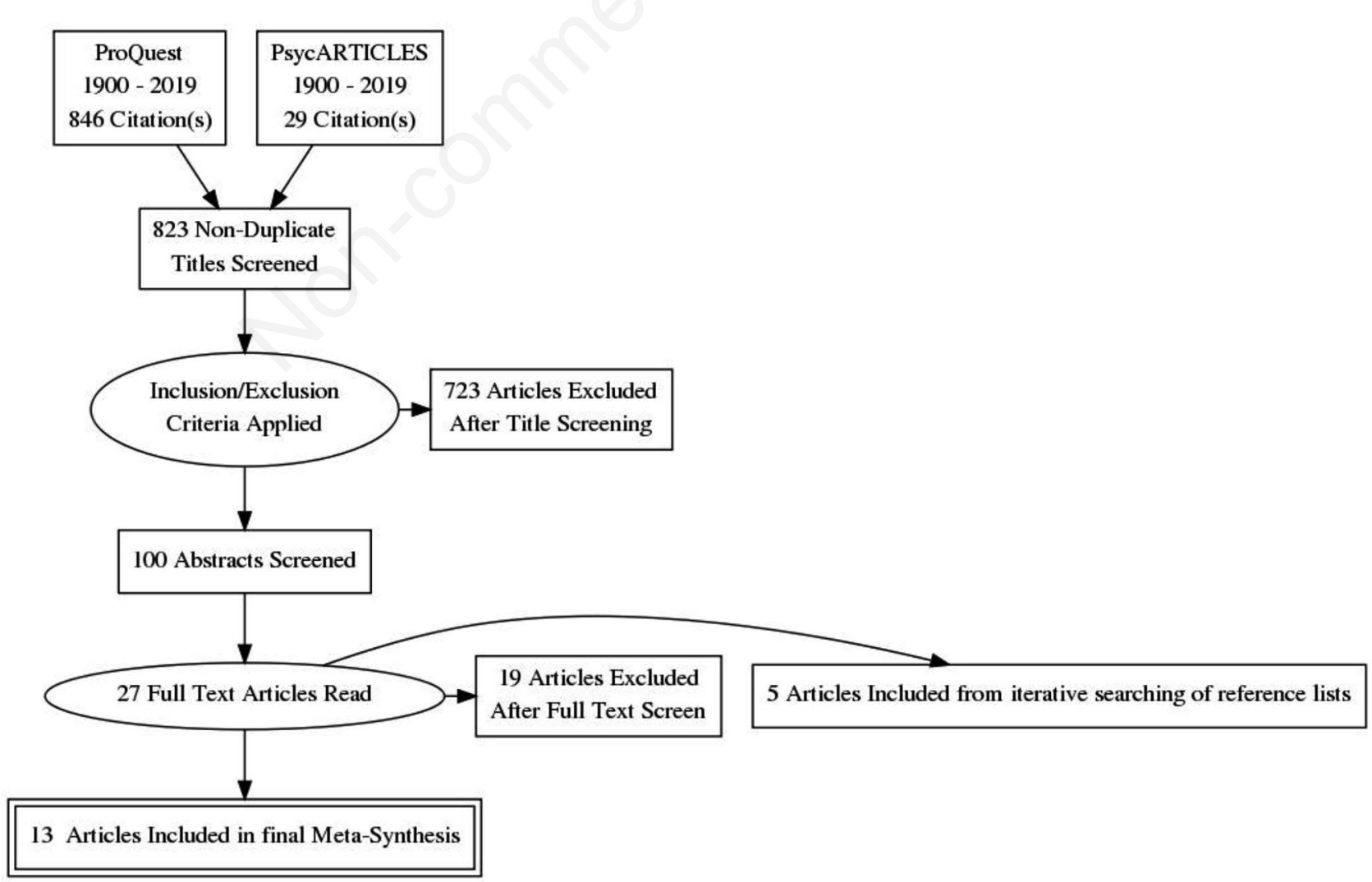

Figure 1. PRISMA diagram demonstrating systematic study selection procedure (PRISMA Flow Diagram Generator, n.d). 
Lack of methodological specification has been noted by other meta-syntheses researchers (i.e., Atkins et al., 2008) without compromising analytic capacity for meta-synthesis. Thus, it is acknowledged that even when study methodologies are inadequately or incorrectly reported, this does not necessarily equate to substandard research (Atkins et al., 2008; Sandelowski et al., 1997).

\section{Analytic procedure}

Noblit and Hare's (1988) meta-ethnographic approach to data analysis was selected for the present meta-synthesis. Arguably the most established approach to conducting meta-syntheses, Noblit and Hare's (1988) method allows for an interpretative and dynamic process of data analysis via an iterative seven-step procedure.

The seven phases of analysis comprise i) identifying a research interest, ii) determining relevant accounts, iii) repeated readings, iv) considering the inter-relationships of studies, v) study translation, vi) synthesising translations, and vii) reporting the final synthesis.

The first two stages were achieved through literature searches as previously outlined. Repeated readings of the selected studies enabled a deep and active engagement with the data. First, second and third order constructs were identified and tabulated to identify emergent themes and concepts. Constant comparison identified complementary patterns, themes or concepts across studies. Findings that were incongruent with the emerging analysis were also considered from the perspective of refutation, providing a representative account of the full dataset. These were expressed in the final synthesis alongside novel interpretations apparent across studies, elevating the analytic contribution.

As there remains a lack of consensus regarding what study information may be considered interpretable data, the present meta-synthesis analysed the complete results sections of published studies. Owing to the considerable variability of presented findings across doctoral theses, all sections in the main text that included raw transcript excerpts were subsequently analysed (primarily findings and discussion chapters). All data were analysed line by line, whereby a single quote could lead to the development of multiple codes, thematic codes, subthemes or the development of larger master concepts.

Three of the selected studies conducted a comparative analysis of the experiences of pregnant therapists with other clinical groups (clients, physicians and expectant adopting therapists respectively; McCluskey, 2017; Matozzo, 2000; Davis, 1997). In these instances, only information pertaining to the experiences of pregnant therapists was included in the analysis.

\section{Results}

While the majority of studies focus on the therapists' experiences during pregnancy, one study primarily explored the post-partum experiences of new mothers returning to therapeutic work (Zackson, 2012). Seven studies employed retrospective interview designs to elicit information pertaining to therapists' pregnancies. Four

Table 1. CASP qualitative checklist for the meta-synthesis demonstrating how each study in the meta-synthesis addresses the CASP qualitative checklist (2017) quality measures.

\begin{tabular}{|c|c|c|c|c|c|c|c|c|c|c|}
\hline & \multicolumn{10}{|c|}{ CASP Checklist Questions } \\
\hline & 1 & 2 & 3 & 4 & 5 & 6 & 7 & 8 & 9 & 10 \\
\hline Bashe (1989) & $\checkmark$ & $\checkmark$ & $\checkmark$ & $\checkmark$ & $\checkmark$ & $?$ & $?$ & $?$ & $\checkmark$ & $\checkmark$ \\
\hline Baum (2006) & $\checkmark$ & $\checkmark$ & $\checkmark$ & $\checkmark$ & $\checkmark$ & $\mathrm{X}$ & $?$ & $\checkmark$ & $\checkmark$ & $\checkmark$ \\
\hline Baum (2010) & $\checkmark$ & $\checkmark$ & $\checkmark$ & $\checkmark$ & $\checkmark$ & $\mathrm{X}$ & ? & $\checkmark$ & $\checkmark$ & $\checkmark$ \\
\hline Byrnes (2001) & $\checkmark$ & $\checkmark$ & $\checkmark$ & $\checkmark$ & $\checkmark$ & $?$ & ? & $\checkmark$ & $\checkmark$ & $\checkmark$ \\
\hline Davis (1997) & $\checkmark$ & $\checkmark$ & $\checkmark$ & $\checkmark$ & $\checkmark$ & $\checkmark$ & $\checkmark$ & $\checkmark$ & $\checkmark$ & $\checkmark$ \\
\hline Fenster (1983) & $\checkmark$ & $\checkmark$ & $\checkmark$ & $\checkmark$ & $\checkmark$ & $\mathrm{X}$ & $?$ & $\checkmark$ & $\checkmark$ & $\checkmark$ \\
\hline Grossman (1990) & $\checkmark$ & $\checkmark$ & $\checkmark$ & $\checkmark$ & $\checkmark$ & $\checkmark$ & $?$ & $?$ & $\checkmark$ & $\mathrm{X}$ \\
\hline Kariv-Agnon (1988) & $\checkmark$ & $\checkmark$ & $\checkmark$ & $\checkmark$ & $\checkmark$ & $\mathrm{X}$ & $?$ & $\checkmark$ & $\checkmark$ & $\checkmark$ \\
\hline Locker-Forman (2005) & $\checkmark$ & $\checkmark$ & $\checkmark$ & $\checkmark$ & $\checkmark$ & $\checkmark$ & $?$ & $\checkmark$ & $\checkmark$ & $\checkmark$ \\
\hline Lyndon (2013) & $\checkmark$ & $\checkmark$ & $\checkmark$ & $\checkmark$ & $\checkmark$ & $X$ & $\checkmark$ & $\checkmark$ & $\checkmark$ & $\checkmark$ \\
\hline Matozzo (2000) & $\checkmark$ & $\checkmark$ & $\checkmark$ & $\checkmark$ & $\checkmark$ & $\mathrm{X}$ & $?$ & $?$ & $\checkmark$ & $?$ \\
\hline McCluskey (2017) & $\checkmark$ & $\checkmark$ & $\checkmark$ & $\checkmark$ & $\checkmark$ & $\mathrm{X}$ & $\checkmark$ & $\checkmark$ & $\checkmark$ & $\checkmark$ \\
\hline Zackson (2012) & $\checkmark$ & $\checkmark$ & $\checkmark$ & $\checkmark$ & $\checkmark$ & $\checkmark$ & $?$ & $\checkmark$ & $\checkmark$ & $\checkmark$ \\
\hline
\end{tabular}

$\checkmark$ indicates that the researcher has appraised the study as possessing the specified quality aspect; $\mathrm{X}$ indicates that the researcher has appraised the study as lacking the specified quality aspect; ? indicates that the researcher has been unable to sufficiently appraise whether the study may possess the specified quality aspect. 
studies interviewed therapists prospectively, with at least two interview points during pregnancy and post-partum (Byrnes, 2001; Fenster, 1983; Grossman, 1990; LockerForman, 2005), providing a wider range of experiences and limiting recall bias. Six studies reporting the experiences of therapists up to one-year post-partum (Baum, 2006; 2010, interviewed five therapists during third trimester, and five up to one-year post-partum). Additionally, three studies document longitudinal experiences of post-partum therapists up to three, five, and more than ten years, respectively (Matozzo, 2000; McCluskey, 2017; Lyndon, 2013), reducing sample homogeneity.

\section{Demographic characteristics}

The present meta-synthesis reports the cumulative experiences of 157 therapists across thirteen studies (Appendix Table A1). Study publication spans 34 years (1983-2017), with research primarily conducted in North America $(n=11)$ and two linear studies originating in Israel (Baum, 2006; 2010). Variance in reporting therapist demographics was identified, with some studies providing limited information, precluding clear appraisal of the represented therapist population. The extracted frequencies below therefore reflect the available study data as opposed to an inclusive overview. Two published studies used the same set of pregnant therapists as participants (Baum, 2006; 2010); therefore, only data pertaining to Baum (2006) is included in the demographic information. The total demographic study information is thus comprised from twelve of the thirteen included studies.

Most studies interviewed therapists about their experience of their first pregnancy $(\mathrm{n}=119)$, most commonly during the third trimester $(n=88)$. Nine studies provided details of therapists' ages, with a collective age range between 25 and 52 years. Seven studies detailed the experience level of therapists, with an overall range between 0-14 years. Seven studies recruited psycho-dynamically or psychoanalytically trained therapists $(\mathrm{n}=89)$, with re- maining studies reporting that therapists practised a variety of eclectic or integrative approaches. While the majority of therapists worked with adult populations, a smaller proportion exclusively or additionally worked with children and young people $(n=49)$, or groups $(n=12)$. Therapist professional backgrounds included psychologists $(n=87)$, social workers $(n=40)$, psychiatrists $(n=6)$ and other related professions $(\mathrm{n}=6)$. The experiences of 27 pregnant therapists enrolled in training programmes were represented across seven studies (Bashe, 1988; Baum, 2006; 2010; Byrnes, 2001; Locker-Forman, 2005; Lyndon, 2013; Zackson, 2012).

\section{Meta-synthesis findings}

For the present meta-synthesis, four key concepts emerged: Identity Changes, Pregnancy necessitates Disclosure, Therapeutic Challenges and Guilt. The relative endorsement of the four key concepts across the thirteen original studies is demonstrated in Table 2 .

\section{Key Concept 1: Identity Changes}

A highly prevalent theme described by psychotherapists in twelve studies reflects the emerging awareness that pregnancy signifies the development of a new identity: self as mother. Therapists' descriptions indicated that the mother identity was frequently perceived as being in conflict with the therapists' professional identity. This conflict was most pronounced in trainee therapists, whose identities as professionals seemed comparatively underdeveloped, leading to feelings of loss and role ambiguity. Pregnancy also led to a re-evaluation of therapists' relationship with clients, with many relinquishing maternal feelings for clients in favour of their unborn child.

Most therapists spoke excitedly of pregnancy, whilst also expressing apprehension that motherhood may alter their existing self-concept. For some, prospective motherhood represented a "big, big identity" (Davis, 1997; p.70), with the potential to eclipse existing identities. Therapists

Table 2. Study endorsement of the key concepts expressed in the meta-synthesis.

Bash Baum Baum Byrnes Davis Fenster Grossman Kariv- Locker- Lyndon Matozzo McCluskey Zackson (1989) (2006) (2010) (2001) (1997) (1983) (1990) Agnon Forman (2013) (2000) (2017) (2012) (1988) (2005)

\begin{tabular}{|c|c|c|c|c|c|c|c|c|c|c|c|c|c|}
\hline $\begin{array}{l}\text { Key Concept } 1: \\
\text { Identity Changes }\end{array}$ & $\checkmark$ & $\checkmark$ & $\checkmark$ & $\checkmark$ & $\checkmark$ & $\checkmark$ & $\checkmark$ & $\checkmark$ & $\checkmark$ & $\checkmark$ & & $\checkmark$ & $\checkmark$ \\
\hline $\begin{array}{l}\text { Key Concept 2: } \\
\text { a) Telling }\end{array}$ & $\checkmark$ & & & $\checkmark$ & $\checkmark$ & $\checkmark$ & $\checkmark$ & $\checkmark$ & $\checkmark$ & & $\checkmark$ & $\checkmark$ & $\checkmark$ \\
\hline $\begin{array}{l}\text { Key Concept 2: } \\
\text { b) Supervisory Relationships }\end{array}$ & $\checkmark$ & & & & $\checkmark$ & $\checkmark$ & $\checkmark$ & $\checkmark$ & $\checkmark$ & & & & $\checkmark$ \\
\hline $\begin{array}{l}\text { Key Concept 3: } \\
\text { Therapeutic Challenges }\end{array}$ & $\checkmark$ & $\checkmark$ & & $\checkmark$ & $\checkmark$ & $\checkmark$ & $\checkmark$ & $\checkmark$ & $\checkmark$ & $\checkmark$ & $\checkmark$ & $\checkmark$ & $\checkmark$ \\
\hline $\begin{array}{l}\text { Key Concept 4: } \\
\text { Guilt }\end{array}$ & $\checkmark$ & $\checkmark$ & $\checkmark$ & & & $\checkmark$ & $\checkmark$ & $\checkmark$ & $\checkmark$ & $\checkmark$ & $\checkmark$ & & $\checkmark$ \\
\hline
\end{tabular}


reported concerns that they may be unable to maintain both professional and mother identities concurrently, depicting the two as competing drives (Bashe, 1989; Baum, 2010; Davis, 1997; Lyndon, 2013). For some, this constituted a "horrible identity crisis" (Grossman, 1990; p.65) whereby one's professional identity was markedly impacted, or stood to be lost entirely (Bashe, 1989; Baum, 2006; 2010; Davis, 1997; Fenster, 1983; Grossman, 1990; KarivAgnon, 1988; Locker-Forman, 2005; Lyndon, 2013; Zackson, 2012). Themes of loss were apparent in therapists' narratives, whereby pregnancy was considered a transitional stage between professional forfeiture and complete attainment of the mother identity (Davis, 1997; Grossman, 1990). For therapists who were undertaking professional training or had recently qualified, the prospect of losing one's professional identity was especially pronounced. As one trainee reflected: "I don't even know what it feels like to be a therapist. I only know what it feels like to be a pregnant therapist" (Locker-Forman, 2005; p.85), indicating that pregnancy may interfere with role assimilation. Professional curtailment was another shared concern for trainees (Bashe, 1989), whereas for more experienced psychotherapists and second-time mothers (Byrnes, 2001), motherhood represented a temporary hiatus from professional advancement that could be recaptured (Lyndon, 2013; Zackson, 2012) and thus, did not produce the same identity concerns.

Pregnancy also led to a revision in therapists' perceptions of their clinical role (Zackson, 2012), with many identifying that the maternal and protective feelings previously held towards clients had been largely or entirely redirected towards their unborn child (Fenster, 1983; Grossman, 1990; Kariv-Agnon, 1988; Locker-Forman, 2005). This distinction was particularly notable for child psychotherapists and appeared to signify the loss of a special therapeutic alliance: "It's almost like I'm playing mommy, and now I'm going to be a real mommy...I feel less that she's my child" (Locker-Forman, 2005; p.70). Conversely, a few adult therapists indicated that pregnancy increased maternal transference (Byrnes, 2001; Davis, 1997; McCluskey, 2017).

Pregnancy and motherhood were considered to increase identification with and appreciation for clients who were parents, with some considering that their new identity afforded them additional professional credibility (Byrnes, 2001; Locker-Forman, 2005; Zackson, 2012). Simultaneously, therapists reported reduced tolerance for clients who employed abusive or substandard parenting practices (Byrnes, 2001; Zackson, 2012). Several therapists reported increased difficulty encountering child abuse in their work (Byrnes, 2001; Locker-Forman, 2005), finding such themes "very triggering" (Lyndon, 2013; p.93) and increasing the propensity to refer such clients onwards (Zackson, 2012). Some therapists reflected that these difficulties were due to newfound awareness of the vulnerability of children (Locker-Forman,
2005), feeling ineffectual, or over-identification with their own child (Locker-Forman, 2005; Lyndon, 2013; Zackson, 2012).

\section{Key Concept 2: Pregnancy necessitates Disclosure}

Therapists across ten studies discussed the issue of pregnancy disclosure. One striking feature of primiparous therapists' accounts relates to the tendency to defer pregnancy disclosure until clients broach the topic. While this may reflect maintenance of the psychoanalytic blank screen, pregnancy necessitates a clear violation of the therapists' anonymity. Further, compared to the management of other therapeutic changes for which the therapist retains responsibility, discussion of pregnancy appears to be qualitatively distinct. It appears to be placed back with the client, representing a source of considerable anxiety, confusion and guilt. Therapists' regrets regarding disclosure appear linked to the lack of clinical guidance and supervision, which is further discussed in the subsequent subtheme.

\section{Subtheme 1: Telling}

The majority of therapists reported disclosing pregnancies during the second and third trimesters (Bashe, 1989; Byrnes, 2001; Davis, 1997; Fenster, 1983; Matozzo, 2000), with later disclosures associated with therapist regret and poorer clinical outcomes. Of those who announced their pregnancies during the third trimester, many cited delaying disclosures to provide clients with sufficient opportunity to articulate their observations directly (Bashe, 1989; Byrnes, 2001; Davis, 1997; Fenster, 1983; Grossman, 1990; Matozzo, 2000), or via derivatives (a psychoanalytic term referring to subconscious awareness of a phenomenon; Bashe, 1989; Fenster, 1983; Locker-Forman, 2005). Although this provided some valuable opportunities to explore clients' perceptions: "I'm glad I didn't just jump to tell straight away because I don't know that some of the material would have emerged if I had disclosed prematurely" (McCluskey, 2017; p.4); many therapists encountered an impasse whereby clients did not raise the issue (Bashe, 1989; Byrnes, 2001; Davis, 1997; Fenster, 1983; Grossman, 1990; Locker-Forman, 2005; Matozzo, 2000). Although some therapists acknowledged that social and therapeutic etiquette might have prevented clients from broaching the subject (Bashe, 1989; Fenster, 1983; Grossman, 1990), others considered this "odd" (McCluskey, 2017; p.4) and necessitated therapists initiating the conversation: "I had to tell her at 7 months. I couldn't believe [the client] hadn't said anything. I was bigger than a house. It was so obvious" (Matozzo, 2000; p.53). This reticence regarding pregnancy disclosure marks a deviation from therapists' standard practice of raising changes to therapeutic arrangements, leaving limited opportunity to explore the pregnancy therapeutically: "I probably only had three sessions before I was set to leave" (Byrnes, 2001; p.169). 
Conversely, prompt disclosures were reported by multiparous and more experienced therapists (Byrnes, 2001; Davis, 1997; Locker-Forman, 2005) and those initiating therapy with new clients, indicating that factors separate from therapeutic orientation may influence disclosure decisions. Additional factors reported to affect the timing of disclosures included awareness that the pregnancy was showing (Byrnes, 2001), to prevent the client learning of the pregnancy via alternative channels (Bashe, 1989) and client formulations (Byrnes, 2001; Fenster, 1983; Grossman, 1990).

Pregnancy was emotionally complex for therapists and produced an array of affective experiences; some described sharing as a "relief" (Grossman, 1990; p.74), and explained that withholding felt "secretive" (McCluskey, 2017; p.4), "unnatural" (Grossman, 1990; p.66) and like "a betrayal" (Byrnes, 2001; p.150). However, the majority reported that disclosure was difficult and anxiety provoking (Bashe, 1989; Byrnes, 2001; Davis, 1997; Kariv-Agnon, 1988; Matozzo, 2000; Zackson, 2012) due to fears of inciting client anger (Bashe, 1989; Matozzo, 2000) or causing therapeutic ruptures (Bashe, 1989; Matozzo, 2000). For several therapists, the act of disclosure was guilt inducing: "I felt like I was betraying her" (Locker-Forman, 2005; p.80). Resultantly, therapists often planned disclosures carefully (Kariv-Agnon, 1989; Matozzo, 2000;), while others attempted to conceal their pregnant form (Locker-Forman, 2005), or did not disclose at all (Davis, 1997; Fenster, 1983; Locker-Forman, 2005).

In retrospect, therapists acknowledged shortcomings related to their pregnancy disclosures, with some voicing concerns that it had been poorly managed (Bashe, 1989; Kariv-Agnon, 1988; Locker-Forman; 2005). Many cited late disclosure as the key factor for client dropout: "because we did not deal with the pregnancy the case ended" (Locker-Forman, 2005; p.78-79). In addition to disclosing earlier (Byrnes, 2001; Fenster, 1983; Locker-Forman; 2005), therapists reflected that future pregnancies could be more effectively managed by being more "direct" (Fenster, 1983; p.53), anticipating personal questions (Byrnes, 2001; Kariv-Agnon, 1988) and probable client reactions in advance (Zackson, 2012); alongside ensuring that clients learn of the pregnancy directly from the therapist (Byrnes, 2001).

\section{Subtheme 2: Supervisory Relationships}

One conspicuously absent feature across seven studies relates to the lack of supervisory advice regarding pregnancy. Further, where guidance was received this was clinically inauspicious, advocating postponed disclosure. As previously outlined, late disclosure was associated with poorer therapeutic outcomes and therapist regret, emphasising the limited utility of supervision. The non-exploratory approach to pregnancy taken by supervisors was mirrored in therapists' subsequent client interactions. Therapists' desire for liaison with formerly pregnant col- leagues and supervisors reflects the need for sources of professional identification, especially when supervision does not consider the therapeutic impact of pregnancy.

Even when supervisors were highly regarded and skilled at managing other clinical issues, supervision was largely perceived as inadequate with regard to managing the therapeutic impact of pregnancy (Bashe, 1989; Fenster, 1983; Kariv-Agnon, 1988; Locker-Forman, 2005). Pregnancy was purported to instigate supervisory role reversals such as reassurance seeking (Fenster, 1983) and trigger personal issues for supervisors (Locker-Forman, 2005; Zackson, 2012). Some therapists expressed regret for following supervisory direction regarding disclosure: "the supervisor had no advice except don't talk about it, don't talk about it, don't talk about it. Wait for it, wait wait wait. And that was a mistake" (Locker-Forman, 2005; p.79). Indeed, some therapists who were disinclined to discuss their pregnancies reported high levels of attrition (Fenster, 1983; Locker-Forman, 2005; Zackson, 2012), highlighting possible clinical implications.

Sometimes therapists' interactions with clients appeared broadly to reflect the tone of supervisory exchanges. For example, one therapist detailed her supervisor's reluctance to explore her pregnancy: "I feel her uncomfortableness which makes me want to back away from it" (Fenster, 1983; p.113), which was in turn mirrored by her own disinclination to discuss the pregnancy with clients. Another therapist who was "furious" at her supervisor's perfunctory response to a client's threat to kill her baby, described the possible impact of having a similarly cursory exchange with her client: "the patient's aggression wasn't handled well...if she was allowed by me and others to express [hate], the aggression wouldn't have become this destructive" (Zackson, 2012; p.94). Others described the same parallel process occurring in reverse, whereby therapists with supportive supervisory relationships reported forging stronger therapeutic alliances with clients during pregnancy (Zackson, 2012). While it is not possible to infer causation, this suggests that pregnancy may enhance therapists' sensitivity to countertransference, increasing the need for positive supervisory role models. Indeed, therapists who reported supportive supervisory relationships found that effective containment (Davis, 1997; Lyndon, 2013), alongside having permission to explore how motherhood may affect their therapeutic role, "made a huge difference" (Lyndon, 2013; p.102).

Therapists reported discomfort discussing their pregnancies with male or childless supervisors (Bashe, 1989; Fenster, 1983; Zackson, 2012), who were widely perceived as unsympathetic to the therapeutic challenges encountered during pregnancy. Indeed, some therapists chose to disengage from supervision whilst pregnant (Fenster, 1983). Widely, therapists voiced a general preference for female supervisors with lived experience of providing therapy while pregnant (Fenster, 1983; Lyndon, 
2013; Zackson, 2012), or liaison with formerly pregnant colleagues (Bashe, 1989; Davis, 1997; Fenster, 1983; Kariv-Agnon, 1988; Zackson, 2012); which may indicate that pregnancy enhances therapists' need for sources of professional identification. Supervisory self-disclosures made supervisors more "real" (Fenster, 1983; p.44) and were perceived as helpful for identifying personal limits and boundaries (Baum, 2010), as was practical advice on how to manage changing capabilities during pregnancy, such as floor-based play therapy (Locker-Forman, 2005). While some trainee therapists reflected on the utility and supportive nature of supervision (Lyndon, 2013), others reported discomfort with the pregnancy taking an increasingly central role in supervisory discussions: "supervision was actually sometimes an experience of therapy at the expense of supervision" (Baum, 2006; p.567). While the cause of this discrepancy remains unknown, this may suggest that frequent evaluation alongside a lesser-developed professional identity, may increase trainees' reluctance to discuss topics perceived as personal.

Attempts to substitute or supplement supervision with literature were reported to be unprofitable (Davis, 1997; Fenster, 1983). Similarly, therapists' core training was considered insufficient at preparing therapists to manage client reactions to pregnancy (Zackson, 2012), leaving some feeling "lost" (Zackson, 2012; p.108) and "completely unprepared" (Bashe, 1989; p.74). Indeed, therapists reported that study participation either represented the first opportunity to explore the impact of pregnancy (Kariv-Agnon, 1988) or a more intensive examination than clinical supervision, identifying outstanding therapeutic issues (Byrnes, 2001; Fenster, 1983; Zackson, 2012). The dearth of available guidance was perhaps best illustrated by one therapist who requested the study researcher's advice regarding a client's request to meet her baby: "What should I do? What would you do?" (Zackson, 2012; p.91). Consequently, therapists articulated a need for improved supervisory training (Bashe, 1989; Fenster, 1983) or specialised consultation to address pregnancy related clinical issues (Byrnes, 2001; Davis, 1997).

\section{Key Concept 3: Therapeutic Challenges}

Therapists in twelve studies encountered significant and unfamiliar therapeutic challenges. Strikingly, therapists were frequently subjected to highly threatening and emotionally charged material related to pregnancy, increasing therapist vulnerability. Without adequate supervision, therapists lack a forum to emotionally process this material, leading to increased stoicism and the loss of therapeutic curiosity. Conversely, pregnancy also precipitated increased personal questions from clients. For most, fidelity to the blank screen was futile, whereas enhanced personal disclosure was therapeutically advantageous. Pregnancy therefore appears to necessitate unconventional working practices, which therapists are largely attempting to navigate alone.
In recognition of the therapeutic disruptions caused by pregnancy, most therapists endeavoured to explore clients' affect and references to expectancy (Bashe, 1989; Byrnes, 2001; Fenster, 1983; Kariv-Agnon, 1988; Matozzo, 2000; McCluskey, 2017; Zackson, 2012). Those who were able to facilitate conversations described pregnancy exploration as "really powerful" (McCluskey, 2017; p.7) and "productive" (Kariv-Agnon, 1988; p.56); which often unearthed new therapeutic material such as clients' own experiences of pregnancy (Davis, 1997; Matozzo, 2000; McCluskey, 2017) and being mothered (Davis, 1997; Fenster, 1983; Kariv-Agnon, 1989). Enhanced openness regarding pregnancy appeared to be related to positive client outcomes (Byrnes, 2001). Conversely, therapists expressed frustration and regret when pregnancy led to the inhibition of client affect (Bashe, 1989; Fenster, 1983; Kariv-Agnon, 1989; McCluskey, 2017; Zackson, 2012) or they were unable to stimulate exploration (KarivAgnon, 1989) with some attributing non-exploration to premature dropout and behavioural escalations (Fenster, 1983; Zackson, 2012).

One area that therapists universally left unexplored was threats of infanticide (Bashe, 1989; Davis, 1997; Fenster, 1983; Grossman, 1990; Kariv-Agnon, 1988; LockerForman, 2005; Zackson, 2012). Understandably, therapists reported extreme difficulty listening to clients' fantasies of harm and miscarriage, which left them feeling highly distressed, vulnerable and angry for being subjected to such "dangerous, threatening material" (Fenster, 1983; p.92). Similarly, therapists reported aversion to themes of baby loss (Bashe, 1989; Kariv-Agnon, 1988; Locker-Forman, 2005), which often arose spontaneously in response to pregnancy disclosure. Therapists described feeling upset by the insensitivity of clients who recounted tales of miscarriage: "I just couldn't handle it... I was mad at her" (Kariv-Agnon, 1988; p.70). The residual impact of such confrontational content was apparent when one therapist cried recounting a client's threats towards her unborn baby (Bashe, 1989). Others reported that the pregnancy became a focal point for clients' anger (Bashe, 1989; Baum, 2006; Byrnes, 2001; Davis, 1997; Fenster, 1983; Grossman, 1990; Kariv-Agnon, 1988; McCluskey, 2017), which left therapists feeling vulnerable and unwilling to explore or challenge the content. Conversely, a minority of therapists expressed frustration when pregnancy led to the inhibition of client anger (Fenster, 1983; McCluskey, 2017). Pregnancy also signaled an increase in sexual issues, and questions from children (Bashe, 1989; Kariv-Agnon, 1988), and especially from male clients (Bashe, 1989; Fenster, 1983; Matozzo, 2000; McCluskey, 2017), for whom pregnancy revealed both the therapists' sexual unavailability (Bashe, 1989; Fenster, 1983; Grossman, 1990) and fantasies of paternity (Bashe, 1989; McCluskey, 2017). The highly personal nature of these conversations left some therapists feeling objectified (Bashe, 1989) and unwilling to further explore clients' 
fantasies, although some came to later regret this (Fenster, 1983). Incongruously, trainee therapists did not report threatening and sexual content, which may reflect reticence to explore clients' feelings regarding pregnancy or possibly reduced client complexity.

While some therapists feared that self-disclosure constituted a breach of therapeutic fidelity (Bashe, 1989; Grossman, 1990), therapists tended to be upfront in answering clients' many questions: "who the father was, who my husband was. Was I going to keep this baby? Did I want this baby? Was it planned?" (Byrnes, 2001; p.96). Colleagues were more frequently consulted than supervisors when deciding what information to disclose (Bashe, 1989; Fenster, 1983; Kariv-Agnon, 1988), further highlighting the limited utility of supervision.

While enhanced disclosure was sometimes perceived as "intrusive" (Grossman, 1990; Locker-Forman, 2005; Zackson, 2012), the majority discovered that disclosure was "meaningful" (Byrnes, 2001; p.193) and "very conducive" (Grossman, 1990; p.73) to the therapeutic alliance. Perhaps related to the perceived changes to therapists' professional identities, increased candour was also reported to facilitate a therapeutic shift whereby therapists felt increasingly "real" (Bashe, 1989; Byrnes, 2001; Fenster, 1983; Grossman, 1990; Lyndon, 2013; McCluskey, 2017; Zackson, 2012), enabling clients to see them as "a person rather than just a therapist" (Zackson, 2012; p.112). By contrast, relational changes were not reported by therapists who chose to deflect clients' questions (Byrnes, 2001).

Therapists reported implementing increasingly active and directive approaches during pregnancy (Bashe, 1989; Byrnes, 2001; Davis, 1997; Fenster, 1983; Grossman, 1990; Kariv-Agnon, 1988; Zackson, 2012), describing their new working practices as more "confronting" (Fenster, 1983; p.101), " blunt" (Byrnes, 2001; p.186) and "less neutral" (Kariv-Agnon, 1988; p.51). Therapists also reported reduced flexibility regarding rearranging appointments (Bashe, 1989; Fenster, 1983; Grossman, 1990) and were less committed to retaining clients at risk of disengagement (Fenster, 1983). Some attributed these changes to increased time imperatives (Bashe, 1989; Fenster, 1983; Kariv-Agnon, 1988; Zackson, 2012), creating greater urgency to stabilise clients prior to maternity leave (Bashe, 1989; Davis, 1997; Zackson, 2012). Of note, therapists tended to retain their active and disclosing position post-partum (Davis, 1997; Fenster, 1983; Grossman, 1990), indicating that pregnancy may lead to marked changes in therapists' working approaches.

Pregnancy also produced other therapeutic challenges including uninvited touching of the therapists' stomach (Byrnes, 2001; Davis, 1997; Grossman, 1990; LockerForman, 2005) and the receipt of baby gifts, which were widely accepted despite customary refusal (Bashe, 1989; Byrnes, 2001; Fenster, 1983; Grossman, 1990). Other therapeutic quandaries, such as clients' requests to see the therapist's sonogram picture (Locker-Forman, 2005), to hold a baby shower (Byrnes, 2001) and babysit (Bashe, 1989; Fenster, 1983) were also reported. With the exception of a few therapists who brought their babies into sessions (Byrnes, 2001; Grossman, 1990), there is little indication as to how therapists managed these clinical dilemmas or whether additional guidance was sought.

\section{Key Concept 4: Guilt}

Therapists in ten studies described experiencing considerable and unrelenting guilt related to pregnancy. Pregnancy was often viewed as signifying the intentional abandonment of clients, often to the exclusion of other therapeutic interpretations, and was especially pronounced in the accounts of child therapists. The interference of pregnancy symptomatology, such as concentration difficulties, was considered indicative of poor performance, further evidencing therapist beliefs that pregnancy held adverse consequences for clients. Without suitable support and containment, therapists demonstrated an increased propensity to make compensatory therapeutic changes and; in the case of child therapists, consider resignation, emphasising the unyielding nature of therapists' guilt.

Therapists' accounts indicated that pregnancy was frequently viewed as evidence of their deliberate "abandonment" (Locker-Forman, 2005; p.80) of clients. To this effect, pregnancy was widely viewed as a choice that had been "wilfully, consciously" (Fenster, 1983; p.89) inflicted upon clients; with therapists readily endorsing the notion that pregnancy was at the "patients' expense" (Bashe, 1989; p.69). For some, pregnancy became a physical manifestation of the differences between therapists' and clients' life experiences (Bashe, 1989; Baum, 2006; Fenster, 1983; Grossman, 1990; Locker-Forman, 2005), heightening therapist guilt: "I have everything and they have nothing" (Fenster, 1983; p.76). This comparison was exacerbated for therapists working with clients for whom pregnancy was especially confronting, such as those struggling with infertility (Bashe, 1989; Davis, 1997; Grossman, 1990; KarivAgnon, 1988; McCluskey, 2017; Zackson, 2012) and looked after children (Locker-Forman, 2005; Zackson, 2012). Resultantly, pregnancy disclosure was sometimes experienced as an admission of guilt: "The session I told them I was pregnant, I didn't even want to charge them, I felt so bad"' (Matozzo, 2000; p.52).

Therapists working across a range of specialisms described pregnancy guilt. However, the magnitude was especially pronounced in the accounts of child therapists. For some, pregnancy necessitated the revision of omnipotent fantasies of being the client's closest ally, thus generating guilt for "abandoning this child to a world where nobody cares" (Locker-Forman, 2005; p.72). This perceived forfeiture of therapists' personal investment caused some to question their professional commitment and aptitude (Kariv-Agnon, 1988; Locker-Forman, 2005). Therapists' guilt was inadvertently further reinforced by 
children's attempts to convince the therapist to stay: "they would be a better child or they would be good" (Fenster, 1983; p.81), or requests to join the therapists' family: "do I take foster care, could I adopt them" (Byrnes, 2001; p.95). Indeed, the intensity of guilt experienced by therapists was reported to impede engagement with children's emotions, increasing the likelihood of client drop out (Locker-Forman, 2005). Additionally, post-partum child work was often perceived as so confronting that therapists sought alternative employment (Byrnes, 2001; LockerForman, 2005; Zackson, 2012), culminating in the fulfilment of clients' abandonment fears: "Ijust couldn't. It was too painful to tolerate" (Locker-Forman, 2005; p.83).

Pregnant therapists described experiencing physical sensations, such as nausea and baby movements, that divided their attention during clinical work and contributed to recent self-perceptions of inadequate performance (Bashe, 1989; Baum, 2006; 2010; Byrnes, 2001; Davis, 1997; Fenster, 1983; Grossman, 1990; Kariv-Agnon, 1988; Locker-Forman, 2005; Lyndon, 2013; Zackson, 2012). This was especially pronounced in trainee therapists, who frequently interpreted their preoccupation as signifying their professional failure (Baum, 2006; 2010). Indeed, during pregnancy, therapists tended to berate their clinical prowess: "pregnant and a bit competent" (Grossman, 1990; p.68), and questioned whether clients would want to resume therapy following maternity leave (Davis, 1997; McCluskey, 2017), highlighting the consequent impact on therapists' identities as professionals. Pregnancy also stimulated role reversals whereby clients tried to look after the therapist, intensifying feelings of guilt and ineffectiveness (Bashe, 1989; Grossman, 1990; Kariv-Agnon, 1988). Interestingly, multiparous therapists reported guilt less frequently than primiparous therapists. While causation cannot be assessed, it is possible that a therapist's second pregnancy may not evoke the same degree of client reactivity, perhaps because therapists are able to demonstrate their continued availability post-partum (Bashe, 1989; Zackson, 2012).

Therapists reported making therapeutic changes in attempts to alleviate pregnancy guilt, including acquiescing to requests for personal information (Fenster; 1983; Kariv-Agnon, 1988; Zackson, 2012), withstanding verbal tirades (Bashe, 1989; Baum, 2006; Davis, 1997; Fenster, 1983; Grossman, 1990; Kariv-Agnon, 1988; McCluskey, 2017) and going "overboard" (Grossman, 1990; p.62) to meet clients' needs. Occasionally, therapists' efforts to prioritise client care were detrimental to their own health, such as conducting sessions whilst feeling faint (Baum, 2006; Locker-Forman, 2005), fearing miscarriage (Byrnes, 2001) or during premature labour (Davis, 1997). Therapists also attempted to negate the impact of pregnancy during maternity leave by arranging surplus interim cover (Byrnes, 2001; Davis, 1997; McCluskey, 2017), maintaining client contact (Bashe, 1989; Byrnes, 2001) or opting for brief leaves of absence (Bashe, 1989; Baum,
2006; Byrnes, 2001; Davis, 1997). Therapists additionally utilised various protective strategies to alleviate pregnancy-related guilt, including minimising clients' difficulties (Grossman, 1990), repressing memories of confronting cases (Locker-Forman, 2005) or denying the impact of pregnancy on clients' treatment (Bashe, 1989; Kariv-Agnon, 1988). The latter approach was notably described by trainee therapists (Baum, 2010; Lyndon, 2013), who tended to "ignore" (Baum, 2010; p.724) their pregnancies and avoid pregnancy exploration with clients; precluding opportunities to gain contrary evidence.

\section{Discussion}

Owing to the considerable proportion of the therapeutic workforce who are female and of childbearing age, this meta-synthesis explored and detailed the widely unreported experiences of pregnant therapists. A meta-ethnographic analysis led to the development of four key concepts, which indicate that pregnancy creates a multitude of novel personal and therapeutic challenges. The meta-synthesis demonstrated that while therapists' clinical experiences were largely congruent, the emotional burden of pregnancy was most pronounced in the accounts of primiparous, trainee and child therapists.

In accordance with wider literature (Imber, 1990; Goldberger et al., 2003; Nadleson, Notman, Arons \& Feldman, 1974), the meta-synthesis identified therapists' avoidance of highly emotive and threatening material. While this may reflect maternal instincts to protect one's baby, this is incongruent with therapeutic conventions of being receptive to all client experiences. Therapists' reduced exploration has been linked to both increased vulnerability and guilt (Lax, 1969; Raphael-Leff, 2004), enhancing therapist likelihood of responding to clients' pregnancy reactions as indisputable facts. Thus, pregnancy may inadvertently "blind the analyst" (Uyehara, Austrian, Upton, Warner \& Williamson, 1995; p.117), and contribute to the fulfilment of therapists' fears of clinical inefficacy. Alongside offering much needed emotional support, supervision may help to identify avoidant therapist tendencies and consider ways to incorporate difficult material for therapeutic gain.

Therapists' assertions that late pregnancy disclosure results in premature client terminations, supports views that disclosure should occur prior to the third trimester (e.g., Bassen, 1998; Goldberger et al., 2003; Uyehara et al., 1995). Early disclosure may be especially warranted for clients who stand to be particularly affected by therapist pregnancy, such as those struggling with infertility, allowing sufficient time to explore pertinent issues. Further, emerging research indicates that clients place responsibility for pregnancy disclosure firmly with the therapist (McCluskey, 2017), demonstrating the need for forthright disclosures and the added value of research exploring clients' experience of therapists' pregnancy. Combined, 
these findings reveal the futility of supervisory advice issued to therapists in the meta-synthesis to maintain psychoanalytic fidelity during pregnancy. Waldman argues that psychoanalytic practitioners have a "clinical mandate" (2003, p.52) to explore the impact of pregnancy and motherhood in therapy. Indeed, the meta-synthesis identified therapists' concerns that they had not managed pregnancy disclosure and exploration "correctly" (Bashe, 1989; p.43), indicating that pregnancy may enhance therapists' desire for didactic supervisory practices. The lack of pregnancy-specific guidance and supervision is a notable finding that is reflected by professional bodies (e.g., BPS, 2017), highlighting that professional issues relating to pregnancy continue to be overlooked. Indeed, if pregnancy is not regularly discussed in supervision as the meta-synthesis suggests, or if therapists receive explicit instruction not to disclose their pregnancies, it is perhaps unsurprising that therapists report pregnancy-related guilt.

Owing to variation in study demographics, it is not possible to determine how many therapists were in receipt of supervision during pregnancy, although there is some evidence to suggest that some therapists discontinued supervision during pregnancy (Fenster, 1983), calling into further question the utility of supervision. The meta-synthesis indicates that therapists express preferences for supervisors who have themselves successfully negotiated pregnancy and motherhood, perhaps due to increased opportunities for role identification. However, Baum and Itzhaky interviewed mother-supervisors and found that attitudes towards pregnant therapist supervisees were "consistently critical and judgemental" (2006; p.33). This may indicate that supervisory difficulties do not diminish through experiential knowledge, highlighting the need for comprehensive training for all supervisors (see Goldberger and colleagues' 2003 guidance for supervising pregnant therapists). Given the potential parallel processes at play during pregnancy (Fenster et al., 1986; Goldberger et al., 2003), it would be advantageous for supervisors to initiate exploratory discussions about pregnancy, which could subsequently serve as templates for therapists' conversations with clients. Indeed, in a book expanding upon Fenster's (1983) clinical dissertation, Fenster, Phillips and Rapoport (1986) suggest that pregnancy exploration should begin in supervision before being extrapolated to the therapy room. However, as the meta-synthesis indicates, it may be beneficial for supervisors to prospectively discuss the clinical rationale for such supervisory modifications, especially with trainee therapists who may otherwise find the introduction of personal content disconcerting.

When considering the meta-synthesis' findings, it is important to remain mindful of the following limitations. Firstly, the breadth of focus of the individual studies varied considerably, impacting how much each paper contributed to the final synthesis, which may have led to some findings being overrepresented. This may be further skewed by the inherent difficulty analysing qualitative studies with small sample sizes, most of which were conducted in North America where maternity leave is often restrictive. Further, the variance in the demographic data provided by studies limits the capacity to draw firm conclusions about the experiences of pregnant therapists, and subsequently to whom the findings may be most applicable. This precludes opportunities for subsequent analyses, such as matched demographic comparisons or exploring the unique contributions of therapist-specific variables. For example, the experiences of group psychotherapists are notably underrepresented. Research exploring the therapeutic experiences of matched pregnant therapist and supervisor dyads would be particularly informative. Comparative studies exploring the therapeutic impact of other sudden changes, including therapist illness or disability, are also warranted. Finally, many of the included studies are notably dated, which may have influenced the reported findings, i.e., prevalence of psychodynamic working practices. However, many findings such as guilt and identity conflicts are reflected across the wider study corpus, indicating the consistency of therapists' experiences over time and the continued lack of adequate therapist support.

\section{Conclusions}

Although pregnancy reflects a brief transitional stage in the careers of therapists, the meta-synthesis indicates that many of the therapeutic changes derived during pregnancy may lead to long-term clinical changes, such as enhanced self-disclosure. While this may be viewed as an assimilative process of identity reformation or reappraisal of job role, it may also be the result of insufficient support during a formative chapter of therapists' careers. Indeed, the meta-synthesis suggests that changes to working practices during pregnancy, such as reduced exploration of client affect, emerge (partially) in response to uncontained therapist guilt. Supervisors may therefore need to be especially vigilant for therapist guilt, especially primiparous child therapists, as for some, pregnancy led to resignation. Plainly, these findings highlight that existing support frameworks and guidelines are insufficient during pregnancy and require urgent attention to promote retention.

\section{References}

Atkins, S., Lewin, S., Smith, H., Engel, M., Fretheim, A., \& Volmink, J. (2008). Conducting a meta-ethnography of qualitative literature: Lessons learnt. BMC Medical Research Methodology, 8(1), 21. doi:10.1186/1471-2288-8-21

Barroso, J., Gollop, C. J., Sandelowski, M., Meynell, J., Pearce, P. F., \& Collins, L. J. (2003). The challenges of searching for and retrieving qualitative studies. Western Journal of Nursing Research, 25(2), 153-178. doi:10.1177/0193945902250034

Bashe, E. D. (1989). The therapist's pregnancy: The experience of patient and therapist in psychoanalytic psychotherapy 
(Doctoral dissertation, Rutgers University). Retrieved from: https://dissexpress.proquest.com/dxweb/results.html?Qry$\mathrm{Txt}=\& \mathrm{By}=\&$ Title $=\&$ pubnum $=9008019$

Bassen, C. R. (1988). The impact of the analyst's pregnancy on the course of analysis. Psychoanalytic Inquiry, 8(2), 280298. doi:10.1080/07351698809533723

Baum, N., \& Itzhaky, H. (2005). Pregnancy as a secret in supervision. Arete, 29(2), 33-43.

Baum, N. (2006). Pregnant field students' guilt. Journal of Social Work Education, 42(3), 561-576. doi:10.5175/JSWE.2006. 200404144

Baum, N. (2010). Dual role transition among first time pregnant social work student trainees. Social Work Education, 29(7), 718-728. doi:10.1080/02615471003599335

Baum, O. E., \& Herring, C. (1975). The pregnant psychotherapist in training: some preliminary findings and impressions. The American Journal of Psychiatry, 132(4), 419-422.

Berman, E. (1975). Acting out as a response to the psychiatrist's pregnancy. Journal of the American Medical Women's Association (1972), 30(11), 456-458.

Bondas, T., \& Hall, E. O. C. (2007). Challenges in approaching metasynthesis research. Qualitative Health Research, 17(1), 113-121. doi:10.1177/1049738306295879

Byrnes, M. J. (2001). The impact of therapist pregnancy on the process of child psychotherapy (Doctoral dissertation, DePaul University) . Retrieved from https://dissexpress.proquest.com/dxweb/results.html?QryTxt=\&By=\&Title=\&pub num $=9982603$

Critical Appraisal Skills Programme (2017). CASP Qualitative Research Checklist. [online]. Retrieved from http://docs. wixstatic.com/ugd/dded87_25658615020e427da194a325e77 73d42.pdf. Accessed: 14th June 2017.

Dyson, E., \& King, G. (2008). The pregnant therapist. Psychodynamic Practice: Individuals, Groups and Organisations, 14(1), 27-42. doi:10.1080/14753630701768958

Davis, J. B. (1997). The expectant therapist: Similarities and differences between pregnant and adoptive-expectant psychotherapists (Doctoral dissertation, The Chicago School of Professional Psychology). Retrieved from https://search.proquest.com/docview/619255233? accountid $=14874$

Fenster, S. L. (1983). Intrusion in the analytic space: The pregnancy of the psychoanalytic therapist. (Doctoral dissertation, Adelphi University). Retrieved from: https://dissexpress. proquest.com $/$ dxweb/results.html? QryTxt $=\& B y=\&$ Title $=\&$ pubnum $=8317555$

Fenster, S., Phillips, S. B., \& Raporport, E. R. G. (1986). The therapist's pregnancy: Intrusion in the analytic space. Analytic Press, Inc, Hillsdale, NJ.

Finfgeld, D. L. (2003). Metasynthesis: The state of the art so far. Qualitative Health Research, 13(7), 893-904. doi:10. 1177/1049732303253462

Gibb, E. (2004). Reliving abandonment in the face of the therapist's pregnancy. Psychoanalytic Psychotherapy, 18(1), 6785. doi:10.1080/14749730410001656525

Goldberger, M., Gillman, R., Levinson, N., Notman, M., Seelig, B., \& Shaw, R. (2003). On supervising the pregnant psychoanalytic candidate. The Psychoanalytic Quarterly, 72(2), 439-463. doi:10.1002/j.2167-4086.2003.tb00137.x

Grossman, H. Y. (1990). The pregnant therapist: Professional and personal worlds intertwine. In: H. Y. Grossman \& N. L. Chester (Eds.), The experience and meaning of work in women's lives. Hillsdale, NJ, Lawrence Erlbaum Associates Inc,
Hannes, K., \& Macaitis, K. (2012). A move to more systematic and transparent approaches in qualitative evidence synthesis: update on a review of published papers. Qualitative Research, 12(4), 402-442. doi:10.1177/1468794111432992

Imber, R. R. (1990). The avoidance of countertransference awareness in a pregnant analyst. Contemporary Psychoanalysis, 26(2), 223-236. doi:10.1080/00107530.1990. 10746656

Kariv-Agnon, E. (1988). The pregnant therapist: The effect of pregnancy on one's experience as a psychotherapist (Doctoral dissertation, Massachusetts School of Professional Psychology).

Katzman, M. A. (1993). The pregnant therapist and the eatingdisordered woman: The challenge of fertility. Eating Disorders: The Journal of Treatment \& Prevention, 1(1), 17-30. doi:10.1080/10640269308248263

Lax F, R. (1969). Some considerations about transference and countertransference manifestations evoked by the analyst's pregnancy. The International Journal of Psycho-Analysis, $50,363$.

Locker-Forman, A. (2005). When real meets pretend: An exploration of the impact of the therapist's pregnancy on child psychotherapy (Doctoral dissertation, City University of New York). Retrieved from: https://dissexpress.proquest. com/dxweb/results.html? QryTxt $=\& B y=\&$ Title $=\& p u b-$ num $=3159230$

Lyndon, L. G. (2013). Pregnancy, motherhood, and career: Negotiating maternal desires and professional ambition (Doctoral dissertation, The Wright Institute). Retrieved from: https://dissexpress.proquest.com/dxweb/results.html?Qry$\mathrm{Txt}=$ pregnant $+\& \mathrm{By}=$ lyndon $\&$ Title $=\&$ pubnum $=3578746$

Matozzo, L. M. (2000). Impact of the therapist's pregnancy on relationships with clients: A comparative study (Doctoral dissertation, Widener University). Retrieved from: https://dissexpress.proquest.com/dxweb/results.html?QryTxt=pregnant $+\& B y=$ Matozzo $\&$ Title $=\&$ pubnum $=9971339$

McCluskey, M. C. (2017). The pregnant therapist: A qualitative examination of the client experience. Clinical Social Work Journal, 45(4), 301-310. doi:10.1007/s10615-0160599-9

McGourty, A. (2013). The pregnant therapist and the psychotic client: A phenomenological understanding of the impact of the therapist's pregnancy on the therapeutic process. European Journal of Psychotherapy and Counselling, 15(1), 1831. doi:10.1080/13642537.2013.763462

Miller, R. D., \& Giffin, J. A. (2019). Parallel pregnancies: The impact on the supervisory relationship and art therapy practice. The Arts in Psychotherapy. doi:10.1016/j.aip.2018.12.007

Mohammed, M. A., Moles, R. J., \& Chen, T. F. (2016). Medication-related burden and patients' lived experience with medicine: A systematic review and metasynthesis of qualitative studies. BMJ Open, 6(2), e010035. doi:10.1136/bmjopen2015-010035

Nadelson, C., Notman, M., Arons, E., \& Feldman, J. (1974). The pregnant therapist. The American Journal of Psychiatry, 131(10), 1107-1111.

Naparstek, B. (1976). Treatment guidelines for the pregnant therapist. Psychiatric Opinion, 13(1), 20-25.

Napoli, M. (1999). Issues for pregnant therapists: Missed appointments and fee payments. British Journal of Psychotherapy, 15(3), 355-367. doi:10.1111/j.1752-0118.1999. tb00459.x

Noblit, G. W., \& Hare, R. D. (1988). Meta-ethnography: Syn- 
thesizing qualitative studies (Vol. 11). London, Sage Publications.

PRISMA (n.d.). PRISMA Flow Diagram Generator. Retrieved from: http://prisma.thetacollaborative.ca/

Sandelowski, M., \& Barroso, J. (2003). Classifying the findings in qualitative studies. Qualitative Health Research, 13(7), 905-923. doi:10.1177/1049732303253488

Sandelowski, M, Docherty, S. \& Emden, C. (1997). Qualitative Metasynthesis: Issues and Techniques. Research in Nursing and Health, 20, 365-372. doi:10.1002/(SICI)1098240X(199708)20:4<365::AID-NUR9>3.0.CO;2-E

Schmidt, F. M. D., Fiorini, G. P., \& Ramires, V. R. R. (2015). Psychoanalytic psychotherapy and the pregnant therapist: A literature review. Research in Psychotherapy: Psychopathology, Process and Outcome, 18(2), 50-61. doi:10.4081/ ripppo.2015.185

Schmidt, F. M. D., Gastaud, M. B., \& Ramires, V. R. R. (2018). Child Psychodynamic Psychotherapy and Therapist Pregnancy: Systematic Case Study. Paideía, 28, n/a. doi:0.1590/1982-4327e2836

Silverman, S. (2001). Inevitable disclosure: Countertransference dilemmas and the pregnant lesbian therapist. Journal of Gay \& Lesbian Psychotherapy, 4(3/4), 45. doi:10.1300/ J236v04n03_04
Tonon, C. B., Romani, P. F., \& Grossi, R. (2012). The therapist's pregnancy and its consequences on the psychotherapeutic process. Psicologia: Teoria E Pesquisa, 28(1), 87-92. doi:10.1590/S0102-37722012000100011

Uyehara, L. A., Austrian, S., Upton, L. G., Warner, R. H., \& Williamson, R. A. (1995). Telling about the analyst's pregnancy. Journal of the American Psychoanalytic Association, 43(1), 113-135. doi:10.1177/000306519504300110

Waldman, J. (2003). New mother/old therapist: Transference and countertransference challenges in the return to work. American Journal of Psychotherapy, 57(1), 52-63. doi:10.1176/appi.psychotherapy.2003.57.1.52

Walsh, D., \& Downe, S. (2005). Meta $\square$ synthesis method for qualitative research: A literature review. Journal of Advanced Nursing, 50(2), 204-211. doi:10.1111/j.1365-2648. 2005.03380.x

Whyte, N. (2004). Review of the Literature. Psychoanalytic Psychotherapy, 18(1), 15-26. doi:10.1080/1474973041000 1656499

Zackson, J. (2012). The impact of primary maternal preoccupation on therapists' ability to work with patients (Doctoral dissertation, City University of New York). Retrieved from: https://search.proquest.com/docview/1353283755/349FB64 20A7B436EPQ/7? accountid=14874 